\title{
articles
}

\section{Factors associated with relapse in schizophrenia}

\author{
N J B Kazadi, MD \\ M Y H Moosa, MMed (Psych), FCPsych, MCFP \\ F Y Jeenah, MMed (Psych) \\ Division of Psychiatry, University of the Witwatersrand, \\ Johannesburg
}

Aim. Early identification and prevention of relapse in patients with schizophrenia has significant therapeutic and socioeconomic implications. The aim of this study was to determine the factors, if any, that may be associated with relapse in a group of patients in Johannesburg.

Method. Patients were recruited from mental health outpatient clinics in a predominantly residential area during the period January 1995 - June 2005. They were included if a review of their records confirmed a diagnosis of schizophrenia according to the Diagnostic and Statistical Manual of Mental Disorders (4th edition) (DSM-IV); they had no other psychotic illness; and they were $\geq 18$ years old. Patients were excluded if the diagnosis of schizophrenia had first been made in the preceding 6 months. Demographic and clinical characteristics of the patients were obtained from their case notes.

Results. Of the 217 patients who were included in the study, $61.8 \%(N=134)$ had a history of at least 1 relapse. There was no significant difference ( $p>0.05)$ between those who relapsed and those who did not relapse in terms of gender, marital status or employment status. Approximately $46 \%(\mathrm{~N}=61)$ of those who relapsed had co-morbid psychiatric disorders, compared with $10.8 \%(N=9)$ in those who did not relapse $(p<0.0001)$, but there was no significant difference between the two groups when comparing the presence of co-morbid medical disorder ( $p=0.348)$. Nearly half $(N=63)$ of patients who relapsed had a history of substance abuse $(p=0.0054)$; cannabis was significantly more abused $(p=0.0014)$. Twothirds ( $N=138$ ) of the study population did not adhere to their treatment, of whom $80.4 \%(N=107)$ experienced a relapse $(p<0.0001)$. Significant multiple logistic regression models for patients who relapsed included poor adherence due to sideeffects (odds ratio $(O R)=3.032 ; p=0.023 ; 95 \%$ confidence interval (CI) 1.168- 7.870); poor adherence due to lack of insight $(O R=5.29 ; p<0.0001 ; 95 \% \mathrm{Cl} 2.28-12.20)$, and co-morbid depressed mood $(O R=5.33 ; p<0.001 ; 95 \% \mathrm{Cl}$ $2.32-12.221$.
Conclusion. Co-morbid depressed mood, poor adherence owing to lack of insight, and medication side-effects were the factors most likely to increase the risk of relapse in patients with schizophrenia. Risk of relapse may be reduced when the treating psychiatrist identifies and addresses these factors.

Schizophrenia is a chronic and disabling illness that affects approximately $1 \%$ of the world's population. It is often accompanied by relapse even while on treatment. 'Relapse rates vary from $50 \%$ to $92 \%{ }^{2}$ and are similar in developed and developing countries, despite the former having well-established mental health services. Among South Africans, there are few published data regarding the prevalence and factors associated with relapses; this study was intended to address that need.

Relapse in schizophrenia is broadly recognised as the reemergence or the worsening of psychotic symptoms. More specifically, certain criteria are used to define relapse; they include aggravation of positive or negative symptoms, hospital admission in the past 6 months, and more intensive case management and/or a change in medication. ${ }^{3}$ Relapse may result in hospitalisation, treatment resistance, cognitive impairment owing to progressive structural brain damage, personal distress, incarceration, and interference with rehabilitation efforts. ${ }^{4}$ Relapse increases the economic burden on health care systems because of its associated morbidity and re-admissions to hospital. Prevention of relapses could have significant therapeutic and socio-economic implications. ${ }^{3,5}$ Internationally, the factors commonly associated with relapse include poor adherence to treatment, substance abuse, co-morbid psychiatric illness, a co-morbid medical and/or surgical condition, stressful life events, and the treatment setting. ${ }^{6,7}$

The medication compliance rate for non-psychiatric illnesses is $76 \%$, while that for psychiatric illnesses is $58 \% .{ }^{8}$ More specifically, about half of the patients with schizophrenia are non-adherent to treatment. 9,10 This non-adherence may be due to factors that are patient-related (e.g. substance abuse, forgetfulness, anxiety about side-effects, inadequate knowledge, lack of insight, lack of motivation, fear of stigma); health care-related (e.g. poor patient/ health care provider relationship, poor services and access to services, poor staff training): ${ }^{11}$ socio-economically-related (e.g. illiteracy, low level of education) ${ }^{12}$ or treatmentrelated (e.g. 
poly-pharmacology, complex treatment regimens). ${ }^{13,14}$ Among South Africans, cultural and social attitudes and belief systems are speculated as common reasons for poor adherence to treatment. $^{15}$

Substance abuse is common among patients with schizophrenia ${ }^{16}$ and can lead to relapse independent of its effects on treatment adherence. The lifetime prevalence is estimated to be as high as $47 \%,{ }^{17}$ with approximately $33 \%$ of patients having an alcohol dependence disorder. ${ }^{18}$ Commonly abused substances include nicotine, alcohol, cannabis and cocaine. In South African studies, alcohol abuse and cannabis abuse were reported as significant factors that contributed to relapse in all mental illnesses, ${ }^{19}$ and cannabis, methaqualone and alcohol abuse as having contributed to relapse in acute psychotic states. ${ }^{20}$

Stressful life events are often associated with the onset of a psychotic relapse, usually in the 3 weeks prior to the relapse. ${ }^{21-23}$ Life stressors may be both internal (e.g. thoughts and feelings) and external (e.g. death of a close relative). ${ }^{24}$ Other stressors include chronic interpersonal stress, poverty, homelessness, criminal victimisation and stigma. Patients with schizophrenia are more sensitive and more susceptible to the negative effects of even minor stressors. Unemployment and the loss of a close family member are reported as significant causes of relapse in South Africa. ${ }^{25}$

Depression in schizophrenia has been associated with higher rates of relapse, poor outcome, impaired functioning, personal suffering and even suicide. ${ }^{26,27}$ Approximately 18 - 55\% of patients with schizophrenia make at least 1 suicide attempt, while $10-13 \%$ of patients succeed in committing suicide.?

The aim of this study was to determine the factors, if any, among patients with schizophrenia that may be associated with relapses. The specific objectives were to compare the demographic and clinical characteristics of a group of patients with schizophrenia who relapsed, with those who did not relapse.

\section{Method}

\section{Study sample}

The researchers approached the psychiatric nursing staff of mental health outpatient clinics in Johannesburg for a list of possible patients with a diagnosis of schizophrenia. Patients from this list were then randomly selected using the card-shuffling technique. Patients were included in the study if a review of their records confirmed a diagnosis of schizophrenia according to DSM
IV criteria ${ }^{7}$ and they had no other psychosis, were $\geq 18$ years old, and had attended the clinics between the period January 1995 and June 2005. Patients were excluded if the diagnosis of schizophrenia had first been made in the preceding 6 months, to avoid any bias in detecting relapses in these patients. The University of the Witwatersrand's Human Research Ethics Committee (HREC) approved the study.

\section{Relapse and adherence criteria}

Relapse was identified in the cases of patients who had documented evidence of either re-emergence or aggravation of psychotic symptoms, a consultation with a psychiatrist and medication change for deterioration of illness, and/or admission to a psychiatric unit in a hospital in accordance with the Mental Health Care Act. ${ }^{3}$ Planned hospital admission for a non-related illness or for special investigations was not deemed to be a relapse.

Adherence to treatment was considered to be poor if there was failure to fill any prescription, refusal to take medication, stopping treatment prematurely, and reports of taking medication at the wrong time and/or incorrect dosage.

Demographic and clinical characteristics of the patients /gender, age, marital status, source of income, highest level of education, substance abuse, presence and type of co-morbid psychiatric illness, presence of co-morbid medical/surgical illness, stressful life events, presence of and type of stressor, insight) that affect adherence were obtained directly from their case notes.

\section{Statistical analysis}

Descriptive statistics were computed as mean and frequencies (count and percentages). The two-tailed paired ttest was used to compare continuous characteristics (age) between the groups. Comparisons between relapse and non-relapse groups (outcome variable) with respect to patient characteristics lage, gender, highest level of education, employment status, poor adherence, substance abuse, co-morbid medical/surgical illness, co-morbid psychiatric illness and psychosocial stressors) were examined by the use of contingency tables (chi-squared test with Yates correction and Fisher's exact test, ORs). The variables showing significant association $(p<0.05)$ in the bivariate analyses were entered into the survey logistic regression to obtain the adjusted ORs. The criterion for removal in the multiple logistic regression analysis was $p>0.05$. All analyses were calculated by the Statistical Package for Social Sciences 10.0 for Windows (SPSS Inc., Chicago, USA). 


\section{Results}

\section{Demographic characteristics}

A total of 217 patients with a diagnosis of schizophrenia were included in the study. Most $(61.8 \%, 134)$ of the study population were patients who relapsed, i.e. had a history of at least 1 relapse. The mean age of patients who relapsed was 42.5 years (standard deviation $(S D)=10.5 ; 95 \% \mathrm{Cl} 40.7$ - 44.3; min. 21 years, max. 75 years) while that for patients who did not relapse was 49.3 years $(S D=13.7 ; 95 \% \mathrm{Cl} 46.3-52.3$; min. 21 years, max. 83 years).

About $30.6 \%$ (41) of patients who relapsed had achieved a primary level of education, and $48.5 \%(65)$ a secondary level of education. By comparison, 15.7\% (13) of the patients who did not relapse had achieved a primary level of education, and $72.3 \%(60)$ a secondary level of education (Fisher's exact $=0.006 ; p=0.01$ ) (Table I). There was no significant difference between the two groups regarding gender (Fisher's exact=1.000, $p=0.98$ ); marital status (Fisher's exact=0.001, $p=0.12$ ); receiving state grants (Fisher's exact $=0.471, p=0.52$ ); receiving family support (Fisher's exact $=0.203, p=0.31$ ); and employment status (Fisher's exact=0.203, $p=0.31$ ).

\section{Clinical characteristics}

Approximately $46 \%(61)$ of patients who relapsed had co-morbid psychiatric disorders (40.2\% had a depressed mood) compared with $10.8 \%(9)$ of patients who had not relapsed ( $p<0.0001$ ) (Table II). Further, 15.2\% (20) of patients who relapsed had either attempted suicide or had suicidal ideation compared with none among those who had not relapsed $(p=0.0002)$. There was no significant difference between the two groups regarding comorbid medical disorders (Fisher's exact=0.286, $p=0.35$ ).

Nearly $50 \%$ (63) of the patients who relapsed had a history of substance use/abuse compared with 30. 1\% (25) of patients who had not relapsed $(p<0.05)$; cannabis was the preferred drug of use/abuse $(p<0.0001)$.

Two-thirds (138) of the study population were not fully adherent to their treatment, of whom 80.4\% (107) had experienced a relapse (Fisher's exact $=0.000, p<0.0001$ ). The factors that were significantly associated with poor adherence included lack of insight (Fisher's exact $=0.000, p<0.0001$ ) and adverse medication effects (Fisher's exact=0.000, $p=0003$ ) (Fig. 1). Poor relationships with a health care provider (Fisher's exact=1.00, $p=0.80$ ) or living great distances from the clinic (Fisher's exact=0.14, $p=0.09$ ) did not appear to be associated with relapses.

\begin{tabular}{|c|c|c|c|}
\hline Variables & $\begin{array}{l}\text { Relapse } \\
N=134 \text { (100\%) }\end{array}$ & $\begin{array}{l}\text { Non-relapse } \\
N=83(100 \%)\end{array}$ & \\
\hline \multicolumn{4}{|l|}{ Gender* } \\
\hline Males & $93(69.4)$ & $56(69.1)$ & \\
\hline Females & $41(30.6)$ & $25(30.8)$ & Fisher's exact $=1.000 ; p=0.98$ \\
\hline \multicolumn{4}{|l|}{ Marital status* } \\
\hline Single & $96(73.3)$ & $49(59.0)$ & \\
\hline Married & $18(13.7)$ & $20(24.1)$ & \\
\hline Divorced & $16(12.2)$ & $13(15.7)$ & \\
\hline Unknown & $1(0.8)$ & $1(1.2)$ & Fisher's exact $=0.001 ; p=0.12$ \\
\hline \multicolumn{4}{|l|}{ Education } \\
\hline No formal & $19(14.2)$ & $8(9.6)$ & \\
\hline Primary & $41(30.6)$ & $13(15.7)$ & \\
\hline Secondary & $65(48.5)$ & $60(72.3)$ & \\
\hline Tertiary & $9(6.7)$ & $2(2.4)$ & Fisher's exact $=0.006 ; p=0.01$ \\
\hline \multicolumn{4}{|c|}{ Source of income ${ }^{\dagger}$} \\
\hline Nil & $4(3.0)$ & $4(4.8)$ & \\
\hline Employed & $9(6.7)$ & $9(10,8)$ & Fisher's exact $=0.203 ; p=0.31$ \\
\hline State grants & $112(83.6)$ & $66(79.5)$ & Fisher's exact $=0.471 ; p=0.52$ \\
\hline Family support & $61(45.5)$ & $30(36.1)$ & Fisher's exact $=0.203 ; p=0.31$ \\
\hline
\end{tabular}


Although stressful life events were not significantly associated with relapses (Fisher's exact $=0.000, p=0.001$ ), patients with relapses had more frequent relationship difficulties (Fisher's exact $=0.08$, $p=0.006$ ) and personal stressors (Fisher's exact=0.018, $p=0.02$ ) compared with patients who had not relapsed (Fig. 2).

Multiple logistic regression models were applied to identify factors that best predicted whether or not a patient could relapse. Significant models for patients included partial adherence due to side-effects $(O R=3.032, p<0.05,95 \% \mathrm{Cl} 1.168-7.870)$; partial adherence owing to impaired insight $1 O R=5.278$, $p<0.0001,95 \% \mathrm{Cl} 2.284$ - 12.198); and co-morbid depressed $\operatorname{mood}(O R=5.328, p<0.001,95 \% \mathrm{Cl} 2.323-12.219)$.

\section{Discussion}

\section{Relapse rates}

The most common outcome of schizophrenia is usually a remitting course with one or multiple relapses in $50-92 \%$ of cases. ${ }^{2}$ Patients on medication have a relapse rate of $40 \%$, while those who discontinue their treatment have a 1 -year relapse rate of $65 \%$ and a 2 -year rate of $>80 \%{ }^{28}$ The patients in this study showed similar high rates of relapse. About two-thirds of the patients had at least 1 relapse, with the majority having $\geq 2$ relapses.

In the management of schizophrenia, psychosocial approaches in addition to antipsychotic drug therapy may result in a significant reduction of relapses compared with using antipsychotic treatment only. ${ }^{28}$ The psychosocial approaches include community programmes /case management, assertive community treatment, foster home care, day treatment centres and psychosocial rehabilitation centres) and individual or family psychotherapeutic approaches (social skills training, personal therapy and psychoeducation). South Africa has very little - if any - in the way of

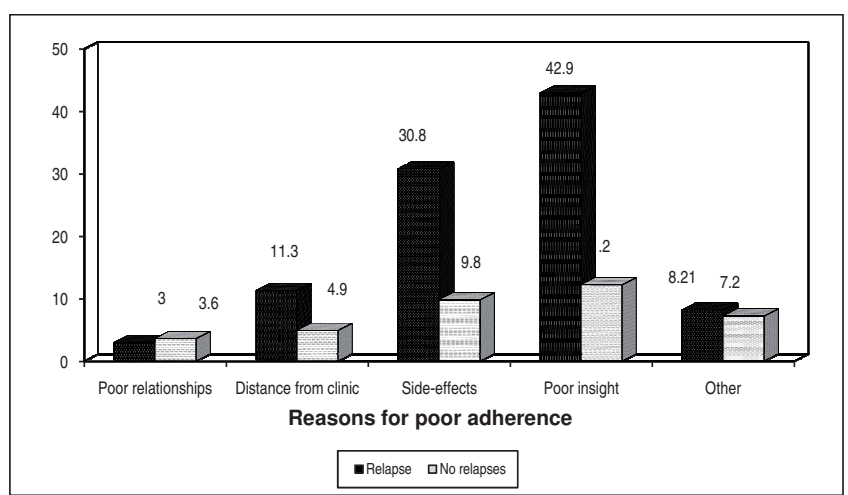

Fig. 1. Reasons for poor adherence in the study population.

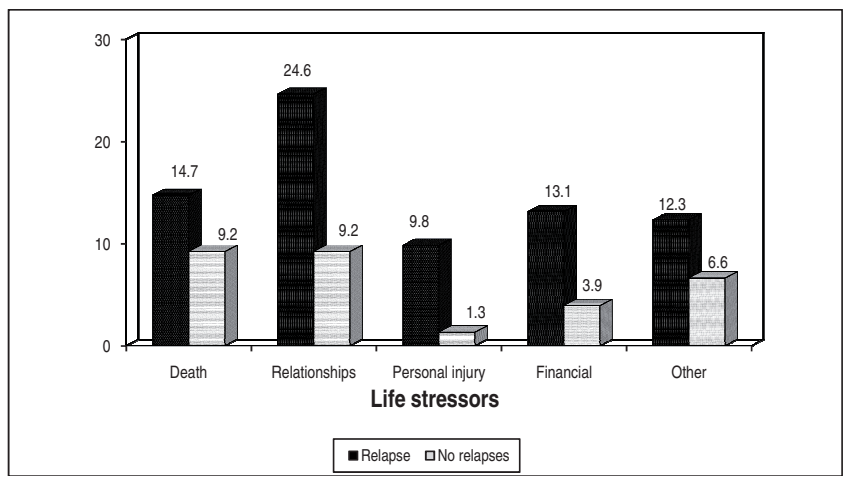

Fig. 2. Life stressors in the study population.

such services available. Patients with schizophrenia are usually discharged from hospitals to follow-up at their nearest community mental health clinics, which focus mainly on pharmacotherapy, with little psychosocial support services owing to a lack of human and material resources ${ }^{29}$ and the difficulties of integrating various treatment modalities in community-based outreach teams - even where the appropriate range of services is available. ${ }^{30}$ Despite these constraints, relapse rates are similar to those of developed countries, notwithstanding the other benefits and qualities that are brought about by psychosocial approaches.

Table II. Clinical characteristics of the study population

\begin{tabular}{|c|c|c|c|}
\hline Variables & $\begin{array}{l}\text { Relapse } \\
N=134(100 \%)\end{array}$ & $\begin{array}{l}\text { Non-relapse } \\
N=83(100 \%)\end{array}$ & \\
\hline Co-morbid psychiatric disorder & $61(45.5)$ & $9(10.8)$ & Fisher's exact $=0.000 ; p<0.05$ \\
\hline Personality disorder & $3(2.3)$ & $2(2.5)$ & Fisher's exact $=1.00 ; p=0.89$ \\
\hline Depressed mood & $53(40.2)$ & $6(7.6)$ & Fisher's exact $=0.000 ; p<0.05$ \\
\hline Suicidal ideation & $20(15.2)$ & $0(0.0)$ & Fisher's exact=0.0; $p<0.05$ \\
\hline Other & $1(0.8)$ & $0(0.0)$ & \\
\hline Co-morbid medical disorder & $43(32.1)$ & $21(25.3)$ & Fisher's exact $=0.286 ; p=0.35$ \\
\hline Substance use/abuse & $63(47.4)$ & $25(30.1)$ & Fisher's exact $=0.000 ; p=0.03$ \\
\hline Poor adherence & $107(80.4)$ & $31(37.8)$ & Fisher's exact=0.000; $p<0.05$ \\
\hline Life stressors & $72(59.0)$ & $18(23.6)$ & Fisher's exact $=0.00 ; p=0.001$ \\
\hline
\end{tabular}




\section{Factors associated with relapses}

\section{Partial adherence}

In mentally ill patients, partial adherence to treatment remains a therapeutic challenge and a factor that is difficult to quantify; it is compounded by the fact that adherence rates vary for different psychotropic medications: 58\% for antipsychotics and 65\% for antidepressants. ${ }^{31}$ We observed an overall adherence rate of $64.2 \%$, which concurs with other reported adherence rates. ${ }^{32-35}$ However, this finding must be qualified in that self-reports of adherence are not always reliable. ${ }^{31}$ For example, patients might not have revealed the extent of their non-adherence for fear of disappointing the clinician. Nevertheless, other methods of assessing adherence (measurement of medication in the blood or urine, clinician rating of response to treatment, pill count, caregiver reports, presence of medication side-effects) also have limitations regarding reliability. It is important to note, furthermore, that poor adherence is not always significantly associated with relapses. $^{2}$

The factors associated with poor adherence to treatment in the diagnostic group under review were medication side-effects and lack of insight. Poor insight contributed to a 5.2-times increase in the risk of relapse - a finding that is consistent with other studies. ${ }^{12}$ However, other researchers argue that the relationship between insight and adherence is not always straightforward and may be without a direct relationship; ${ }^{36}$ e.g. it is possible that the lack of insight in this population might be related to a lower level of formal education and a lack of understanding of mental illness and its phenomena.

Patients who default on their treatment have said that treatment side-effects $^{14,37}$ together with complex treatment regimens ${ }^{38}$ are the main reasons for their poor adherence. The majority of our patients were on typical antipsychotic medication and reported side-effects (mostly extra-pyramidal effects and impotence) as contributing to their poor adherence, which is similar to other reported findings. ${ }^{39}$ However, this association between medication side-effects and adherence is not generally clearly established ${ }^{40,41}$ because many patients adhere to their medication despite treatment side-effects, ${ }^{12}$ and a history of sideeffects is not always predictive of future non-adherence. ${ }^{36}$ Further, recent studies report no added benefits of the atypicals in terms of efficacy, discontinuation rates, adverse effects or quality of life, compared with the typical antipsychotics. ${ }^{32,33,42-45}$

\section{Depressed mood}

A quarter of all patients developed a depressed mood during the period studied, increasing the risk of a relapse by 5.3 times.
Other studies on depression associated with schizophrenia show a variation ranging from a high of $75 \%$ to a low of $7 \%$. ${ }^{26,46}$ Depression symptoms may appear at any time during the course of schizophrenia and contribute to relapse and a decrease in quality of life. ${ }^{47,48}$ However, this is contradicted by Robinson et al. ${ }^{49}$ who reported that despite a possible relationship between mood symptoms and relapse, neither the severity of baseline depressive symptoms nor the presence of mood symptoms was related to relapse and had no prognostic value. Despite these contradictory findings, early detection and management of depression symptoms may decrease the risk of psychotic relapse and it is therefore important that mental health practitioners should identify depressive moods and separate them from the deficit (negative) features of schizophrenia.

\section{Substance abuse}

Although substance abuse was very common among our study population, there was no significant association with relapses. This finding differs from previous findings of strong associations with relapses ${ }^{50-52}$ and a greater risk of re-hospitalisation. ${ }^{53}$ Substance abuse may exacerbate psychotic symptoms, and abused drugs may also lead to transient symptom reduction. ${ }^{54}$ However, some studies report no association between substance abuse and relapse. ${ }^{55}$ It must be emphasised nevertheless that although it is not clear that substance abuse in patients with schizophrenia results in relapse, it does lead to inter alia increased psychosocial problems, infections, sexually risky behaviour, and hostile and disorganised behaviour - and more so in the presence of other health-related problems, poverty, insufficient food, poor housing and widespread unemployment.

\section{Co-morbid medical illnesses}

Surprisingly, only a third of the patients with relapses had comorbid medical illnesses, despite published reports that co-morbid medical disorders exacerbate the relapse process. ${ }^{56,57}$ In South Africa, mental health services remain marginalised and poorly integrated with general medical services in the primary health care system. ${ }^{29,49}$ Co-morbid medical illnesses are managed by different services, and it is possible that adequate attention is not paid to recording these co-morbid medical disorders in case notes at mental health clinics.

\section{Age, education, life stressors and patient-provider relationships}

Other reported factors associated with relapse include age, ${ }^{49,58}$ education, ${ }^{2}$ life stressors and patient-provider relationships. These and other studies, 2,3 failed, however, to confirm these 
associations. The relapses tended to occur in the age group $<50$ years and, although this variable lacked a statistically predictive power of relapse, there was a trend towards younger patients being more likely to relapse. Relapsing patients in this study population were more likely to have achieved only a primary level of education. Schizophrenia tends to have an early age of onset and a chronic course with relapses and a declining level of functioning that contributes to early school drop-out. It is difficult to elucidate a causal relationship between life events and relapse in a retrospective study. The lack of any association between patientprovider relationship and non-adherence should be viewed in the working context; in our setting, doctors change every 6 months. Chronic schizophrenic patients, furthermore, could be compromised by their illness and unable to establish a committed therapeutic alliance with their psychiatrists.

\section{Limitations}

Other reported factors associated with relapses (such as premorbid level of functioning, expressed emotion and duration of untreated psychosis) were not considered in this study and would be better obtained via other study designs. Moreover, the small sample size might have limited our ability to detect statistically meaningful differences.

In any retrospective study design, some data might not have been recorded in case notes, and patients might have been erroneously included or excluded. The design also raises the question of patients' ability to recall events over several weeks between clinic visits, which is compounded by the fact that patients with schizophrenia suffer from some cognitive impairment. However, the majority of our patients' records contained all the data required, and the conclusions drawn are therefore reliable. The study is generalisable, insofar as our study population experiences socio-politico-economic factors and resources that are similar to those experienced by average South African patients with schizophrenia.

\section{Conclusions}

Despite recent therapeutic progress, relapse in schizophrenia is a common and major problem among South Africans. The presence of a co-morbid depressed mood, poor adherence due to a lack of patient insight, and medication side-effects appear to be the factors most likely to increase the risk of a relapse. It is important that, in a local context, the treating psychiatrist identifies and treats mood features along with establishing ways of improving insight and adherence to treatment. If atypical antipsychotic treatments (versus typical antipsychotics) can improve adherence rates, significant reductions in relapse rates and service costs could result.

\section{References}

1. Gelder MG, Lopez-lbor لע, Andreasen NC. New Oxford Textbook of Psychiatry Oxford: Oxford University Press, 2000: 567-621.

2. Suzuki $Y$, Yasumura S, Fukao $S$, et al. Associated factors of rehospitalization among schizophrenic patients. Psychiatry Clin Neurosci 2003; 57: 555-56 1

3. Almond S, Knapp M, Francois C, et al. Relapse in schizophrenia: costs, clinical outcomes and quality of life. Br J Psychiatry 2004; 184: 346-35 1

4. Piggot TA, Carson WH, Saha AR, et al. Aripiprazole for the prevention of relapse in stabilized patients with chronic schizophrenia: a placebo-controlled 26-week study. J Clin Psychiatry 2003; 64: 1048-1057.

5. Knapp M, King D, Pugner K, Lapuerta P. Non-adherence to antipsychotic medication regimens: associations with resource use and costs. Br J Psychiatry 2004; 184: 509516.

6. Harris MG Henry IP. Harrigan SM et al. The relationship between duration of untreated psychosis and outcome: an eight-year prospective study. Schizophr Res 2005; 79: 85-93

7. Diagnostic and Statistical Manual of Mental Disorders - DSM-IV-TR. 4th ed. Washington DC: American Psychiatric Association, 2002

8. Awad GA. Antipsychotic medications: compliance and attitudes towards treatment. Curr Opin Psychiatry 2004; 17: 75-80.

9. Hudson TJ, Owen RR, Trush CR, et al. A pilot study of barriers to medication adherence in schizophrenia. J Clin Psychiatry 2004; 65: 211 1-216.

10. Valenstein M, Blow FC, Copeland LA, et al. Poor antipsychotic adherence among patients with schizophrenia: medication and patient factors. Schizophr Bull 2004 30: 255-264

1 1. Lacro JP, Dunn LB, Dolder CR, et al. Prevalence of and risk factors for medication nonadherence in patients with schizophrenia: a comprehensive review of recent literature. J Clin Psychiatry 2002; 63: 892-908

12. Fenton WS, Blyler CR, Heinssen RK. Determinants of medication adherence in schizophrenia: Empirical and clinical findings. Schizophr Bull 1997; 4: 637-651.

13. Suzuki T, Uchida H, Takeuchi H, et al. Simplifying psychotropic medication regimen into a single night dosage and reducing the dose for patients with chronic schizophrenia. Psychopharmacology 2005; 181: 566-575

14. Perkins DO. Predictors of nonadherence in patients with schizophrenia. J Clin Psychiatry 2002; 63: 12-16

15. Gillis $L S$, Koch A, Joyi M. Improving compliance in Xhosa psychiatric patients. S Afr Med J 1989: 76: 205-208

16. Swofford CD, Kasckow JW, Scheller-Gilkey G, et al. Substance use: a powerful predictor of relapse in schizophrenia. Schizophr Res 1996; 20: 145-151

17. Regier DA, Farmer ME, Rae DS. Comorbidity of mental disorders with alcohol and other drug abuses. JAMA 1990; 264: 2511 1-2518.

18. Salloum IM, Moss HB, Daley DC. Substance abuse and schizophrenia: impediments to optimal care. Am J Drug Alcohol Abuse 1991; 17: 321-336.

19. Solombela PW, Uys LR. Factors influencing the relapse of outpatients with schizophrenia in the Kentani area of Transkei. Curationis 1994; 17: 24-28.

20. Gillis LS, Sandler R, Jakoet A, Elk R. Readmissions to a psychiatric hospital: Outcome on follow-up. S Afr Med J 1986; 70: 735-739.

21. Pourmand DD, Kavanagh DJ, Vaughan K. Expressed emotion as predictor of relapse in patients with comorbid psychoses and substance use disorder. Aust NZJ Psychiatry 2005; 39: 473-478

22. Compton MT, Weiss PS, West JC, et al. The associations between substance use disorders, schizophrenia-spectrum disorders, and Axis IV psychosocial problems. Soc Psychiatry Psychiatr Epidemiol 2005; 40: 939-946.

23. Murray RM, Castle DJ. Genetic and environmental risk factors for schizophrenia. In: Gelder MG, ed. Oxford Textbook of Psychiatry. Oxford: Oxford University Press, 2000

24. Hunter PE, Storat B. Psychosocial triggers of relapse in persons with chronic mental illness: A pilot study. Ment Health Nurs 1994; 15: 67-72.

25. Mwaba K, Molamu RB. Perceived causes of relapse among a sample of recovering patients at a Mafikeng Hospital. Curationis 1998; $21:$ 55-57.

26. Siris SG. Depression in schizophrenia: Perspective in the era of "atypical" antipsychotic agents. Am J Psychiatry 2000; 157: 1379-1389

27. Tollefson GD, Sanger TM, Lu Y, ef al. Depressive signs and symptoms in schizophrenia. A prospective blinded trial of olanzapine and haloperidol. Arch Gen Psychiatry 1998; 55: 250-258.

28. Hogarty GE, Ulrich RF. The limitations of anti-psychotic medication on schizophrenia relapse and adjustment and the contributions of psychosocial treatment. J Psych Research 1998; 32: 243-250

29. Joubert PM. Community psychiatry in South Africa. SA Psych Rev 2002; 5: 4-6.

30. Carpenter WT. Maintenance therapy of persons with schizophrenia. J Clin Psychiatry 1996; 57: 10-16 
31. Marder SR. Overview of partial adherence. J Clin Psychiatry 2003; 64: 3-9

32. Jones PB, Barnes TRE, Davies $L$, et al. Randomized controlled trial of the effect on quality of life of second- vs first-generation antipsychotic drugs in schizophrenia. Arch Gen Psychiatry 2006; 63: 1079-1087

33. Rosenheck RA. Outcomes, costs, and policy caution: A commentary on the cost utility of the latest antipsychotic drugs in schizophrenia study. Arch Gen Psychiatry 2006; 63: 1074-1076.

34. Lieberman JA, Scott Stroup T, McEvoy JP, et al. Effectiveness of antipsychotic drugs in patients with chronic schizophrenia. New Engl J Med 2005; 353: 1209-1223.

35. Thieda P, Beard S, Ritchter A, Kane J. An economic review of adherence with medication therapy in the treatment of schizophrenia. Psychiatr Serv 2003; 54: 508516.

36. Olfson M, Mechanic D, Hansell S, et al. Predicting medication non adherence after hospital discharge among patients with schizophrenia. Psychiatr Serv 2000; 51 : 215-222.

37. Burton SC. Strategies for improving adherence to second-generation antipsychotics in patients with schizophrenia by increasing ease of use. J Psych Pract 2005; 1 1:369378.

38. Razaly MS, Yahya H. Adherence with treatment in schizophrenia. A drug intervention program in a developing program. Acta Psychiatr Scandinav 1995; 91: 331-335.

39. Leucht S, Barnes TRE, Kissling W, et al. Relapse prevention in schizophrenia with new-generation antipsychotics: A systematic review and exploratory meta-analysis of randomized, controlled trials. Am J Psychiatry 2003; 160: 1209-1222.

40. Schooler NR. Relapse and rehospitalization: comparing oral and depot antipsychotics. J Clin Psychiatry 2003; 64: 14-17

41. Fleishhacker WW, Melse U, Gunther V, et al. Adherence with antipsychotic drug treatment: influence of side-effects. Acta Psychiatr Scand 1994; 89: 11-21

42. Lieberman JA, Stroup TS, McEvoy JP, et al. Clinical Antipsychotic Trials of Intervention Effectiveness (CATIE) Investigation. Effectiveness of antipsychotic drugs in patients with chronic schizophrenia. N Engl J Med 2005; 353: 1209-1223.

43. Lieberman JA. Comparative effectiveness of antipsychotic drugs: A Commentary on Cost Utility of the Latest Antipsychotic Drugs in Schizophrenia Study (CUtLASS 1) and Clinical Antipsychotic Trials of Intervention Effectiveness (CATIE). Arch Gen Psychiatry 2006; 63: 1069-1072.

44. Davis JM, Chen N, Glick ID. A meta-analysis of the efficacy of second-generation antipsychotics. Arch Gen Psychiatry 2003; 60: 553-564.

45. Chakos M, LiebermanJ, Hoffman E, et al. Effectiveness of second-generation antipsychotics in patients with treatment-resistant schizophrenia: a review and metaanalysis of randomized trials. Am J Psychiatry 2001; 158: 51 8-526.
46. Du Preez RR, Griffith WC, Page M. Major depressive disorder as a co-morbid diagnosis in schizophrenia versus the diagnosis of schizoaffective disorder - depressed type. S Afr Psychiatry Rev 2005; 8: 134-139

47. Elbogen EB, Swanson JW, Swartz MS, et al. Medication nonadherence and substance abuse in psychotic disorders: impact of depressive symptoms and social stability. J Nerv Ment Dis 2005; 193: 673-679

48. Birchwood M, Mason R, Macmillan F, et al. Depression, demoralization and control over psychotic illness: a comparison of depressed and non-depressed patients with a chronic psychosis. Psychol Med 1993; 23: 387-395

49. Robinson D, Woerner MG, Ma J, et al. Predictors of relapse following response from a first episode of schizophrenia or schizoaffective disorder. Arch Gen Psychiatry 1999; 56: 241-247.

50. Margolese HC, Carlos Negrete J, Tempier R, et al. A 12-month prospective follow-up study of patients with schizophrenia-spectrum disorders and substance abuse: changes in psychiatric symptoms and substance use. Schizophr Res 2006; 83: 65-75.

51. de Leon J, Diaz FJ. A meta-analysis of worldwide studies demonstrates an association between schizophrenia and tobacco smoking behaviors. Schizophr Res 2005; 76: 135-157.

52. de Leon J. Atypical antipsychotic dosing: the effect of smoking and caffeine. Psychiatr Serv 2004; 55: 491-493

53. Sullivan $G$, Wells $K B$, Morgenstern $H$, Leake B. Identifying modifiable risk factors for rehospitalization: A case control study of seriously mentally ill persons in Mississipi. Am J Psychiatry 1995; 152: 1749-1752.

54. Dixon L, Haas G, Weiden P, et al. Acute effects of drug abuse in schizophrenic patients: clinical observations and patients self-reports. Schizophr Bull 1990; 16: 6979

55. Warner R, Taylor D, Wright J, et al. Substance use among the mentally ill: prevalence, reasons for use, and effects on illness. Am J Orthopsychiatry 1994; 64: 30-39.

56. Fenton WS. Comorbid conditions in schizophrenia. Curr Opin Psychiatry 200 1; 14: $17-23$

57. Green Al, Canuso CM, Brenner MJ, Wojcik JD. Detection and management of comorbidity in patients with schizophrenia. Psychiatr Clin N Am 2003; 26: 115 139.

58. Dolder C, Lacro J, Jeste DV. Adherence to antipsychotic and nonpsychiatric medications in middle-aged and older patients with psychotic disorders. Psychosom Med 2003; 65: 156-162. 